\title{
FIDEDIGNIDADE DE UM QUESTIONÁRIO DE COMPORTAMENTO SEDENTÁRIO EM ESCOLARES
}

\section{Reliability of a sedentary behavior questionnaire in students \\ Fidedignidad de un cuestionario de conducta sedentaria de escolares}

\author{
Eliane Denise Araújo Bacil (OrclD) \\ Universidade Federal do Paraná - UFPR - Curitiba (PR) - Brasil \\ Thiago Silva Piola (OrclD) \\ Universidade Federal do Paraná - UFPR - Curitiba (PR) - Brasil \\ Priscila lumi Watanabe (OrcID) \\ Universidade Federal do Paraná - UFPR - Curitiba (PR) - Brasil \\ Michael Pereira da Silva (Lattes) \\ Universidade Federal do Paraná - UFPR - Curitiba (PR) - Brasil
}

Rosimeide Francisco dos Santos Legnani (OrclD)

Universidade Estadual de Ponta Grossa - UEPG - Curitiba (PR) - Brasil

Wagner de Campos (Lattes)

Universidade Federal do Paraná - UFPR - Curitiba (PR) - Brasil

\section{RESUMO}

Objetivo: Avaliar a fidedignidade de um questionário de comportamento sedentário. Métodos: Estudo transversal realizado com 1.119 escolares, de 9 a 15 anos de idade, de sete escolas públicas de Curitiba, Paraná, Brasil, entre março e dezembro de 2015. O questionário é composto por 13 itens, divididos em cinco aspectos (tempo de tela, educacional, cultural, social e transporte), em que os participantes relatam o tempo gasto em atividades sedentárias (horas e/ou minutos) durante uma semana típica. Para a análise de fidedignidade do questionário, realizaram-se duas aplicações do questionário e o coeficiente de Correlação Intraclasse avaliou a reprodutibilidade, o diagrama de dispersão de Bland Altman, a concordância entre as duas medidas teste-reteste e o alpha de Cronbach, a consistência interna do questionário, adotando-se o nível de significância de 5\%. Resultados: O coeficiente de correlação intraclasse e o alpha de Cronbach apresentaram valores maiores que 0,70, sendo maiores nos dias de semana do que nos fins de semana. As duas aplicações do questionário apresentaram uma diferença média de 228,27 minutos/semana, com intervalo de confiança de 95\%, de 175,86 a 280,68. O limite de concordância variou de 2.015,33 a 1.558,79 minutos/semana. Conclusão: O questionário apresentou fidedignidade satisfatória, podendo ser utilizado para mensurar o comportamento sedentário em escolares de 9 a 15 anos de escolas públicas.

Descritores: Reprodutibilidade dos Testes; Inquéritos e Questionários; Estilo de Vida Sedentário; Estudantes.

\section{ABSTRACT}

Objective: To evaluate the reliability of a sedentary behavior questionnaire. Methods: Cross-sectional study with 1,119 students from 9 to 15 years old from seven public schools in Curitiba, Paraná, Brazil, between March and December 2015. The questionnaire consists of 13 items divided into five aspects (screen time, educational, cultural, social and transportation) in which the participants reported time spent in sedentary activities (hours and/or minutes) in a typical week. For the reliability analysis, two applications of the questionnaire were performed and the intraclass correlation coefficient evaluated the reproducibility, Bland-Altman scatter plot assessed the concordance between the two test-retest measurements, and Cronbach's alpha, the internal consistency of the questionnaire, adopting 5\% as significance level. Results: The intraclass correlation coefficient and Cronbach's alpha presented values greater than 0.70 , being higher on weekdays than on weekends. The two applications of the questionnaire presented a mean difference of 228.27 minutes/week, with 95\% confidence interval of 175.86 to 280.68. The limits of agreement ranged from 2,015.33 to -1,558.79 minutes/week. Conclusion: The questionnaire presented satisfactory reliability and can be used to measure sedentary behavior in students from 9 to 15 years old of public schools.

Descriptors: Reproducibility of Results; Surveys and Questionnaires; Sedentary Lifestyle; Students. 


\section{RESUMEN}

Objetivo: Evaluar la fidedignidad de un cuestionario de conducta sedentaria. Métodos: Estudio transversal realizado con 1.119 escolares entre 9 y 15 años de edad de siete escuelas públicas de Curitiba, Paraná, Brasil, entre marzo y diciembre de 2015. El cuestionario tiene 13 ítems divididos en cinco aspectos (tiempo en la pantalla, educacional, cultural, social y transporte) en el cual los participantes relatan el tiempo gastado en las actividades sedentarias (las horas y/o los minutos) durante una semana típica. Para el análisis de la fidedignidad del cuestionario se realizaron dos aplicaciones del cuestionario. El coeficiente de Correlación Intraclase evaluó la reproductibilidad, el diagrama de dispersión de Bland Altman evaluó la concordancia entre las dos medidas test-retest y el alpha de Cronbach evaluó la consistencia interna del cuestionario adoptándose el nivel de significancia del 5\%. Resultados: El coeficiente de correlación intraclase y el alpha de Cronbach presentaron valores mayores que 0,70, siendo mayores en los días de la semana que en los fines de semana. Las dos aplicaciones del cuestionario presentaron una diferencia media de 228,27 minutos/semana con intervalo de confianza del 95\%, entre 175,86 y 280,68. El límite de la concordancia ha variado entre 2.015,33 y 1.558,79 minutos/semana. Conclusión: El cuestionario presentó la fidedignidad satisfactoria y puede ser utilizado para mensurar la conducta sedentaria de escolares entre 9 y 15 años de escuelas públicas.

Descriptores: Reproductibilidad de los Resultados; Encuestas y Cuestionarios; Estilo de Vida Sedentario; Estudiantes.

\section{INTRODUÇÃO}

O comportamento sedentário pode ser definido como a exposição a atividades com baixo dispêndio energético de até 1,5 equivalentes metabólicos (METs) ${ }^{(1)}$. Esse comportamento tem emergido rapidamente como uma importante questão para a saúde pública, sendo objeto de estudo para diferentes desfechos da saúde(2,3).

Independente do nível de atividade física do indivíduo, o excessivo tempo em atividades sedentárias representa um fator de risco em potencial para a saúde das pessoas, pois é positivamente associado ao aumento do risco de doenças cardiometabólicas, de mortalidade por todas as causas e de uma variedade de problemas fisiológicos e psicológicos ${ }^{(4)}$. O comportamento sedentário é responsável por 38\% da mortalidade por todas as causas (aproximadamente 433 mil mortes/ano) em 54 países ${ }^{(5)}$. Assim, mesmo nos indivíduos que seguem as recomendações de atividades físicas moderadas a vigorosas, o tempo prolongado de comportamento sedentário por si só poderá promover efeitos nocivos à saúde ${ }^{(5)}$.

Nas recomendações atuais, preconiza-se que as crianças e os adolescentes dediquem, no máximo, duas horas por dia às atividades sedentárias ${ }^{(6)}$. Com base nessas recomendações, evidências têm demonstrado uma prevalência elevada de adolescentes expostos de forma excessiva a tal comportamento. De acordo com o estudo transnacional Health Behaviour in School-age Children (HBSC), desenvolvido pela Organização Mundial de Saúde (OMS) e realizado em 42 países da Europa e da América do Norte, no período de 2013-2014, os escolares de 11 anos, 13 anos e 15 anos que assistiam televisão por duas ou mais horas durante a semana representaram $50 \%$, $62 \%$ e $63 \%$ dos escolares, respectivamente ${ }^{(7)}$.

Dados nacionais baseados na Pesquisa Nacional de Saúde do Escolar (PENSE) apontam que o hábito de assistir mais de duas horas de televisão por semana foi referido por, aproximadamente, $60 \%$ dos escolares do $9^{\circ}$ ano, sendo maior na região Sudeste $(62,7 \%)^{(8)}$. Outros estudos apresentam prevalências de comportamento sedentário em torno de 50 a $80 \%(9-11)$.

Como visto, o comportamento sedentário apresenta-se como importante fator relacionado à depreciação da saúde de crianças e adolescentes, sendo muito prevalente nessa fase da vida ${ }^{(9-11)}$. Ao se tratar da promoção da saúde nessa população, a avaliação precisa do comportamento sedentário é essencial para monitorar tendências desse comportamento, examinar os efeitos sobre a saúde e avaliar a eficácia das intervenções. No entanto, os instrumentos utilizados para avaliar o comportamento sedentário normalmente avaliam somente o tempo de tela. Crianças e adolescentes também se engajam em outras atividades sedentárias ${ }^{(12)}$.

Com o intuito de avaliar o comportamento sedentário global de adolescentes australianos, pesquisadores desenvolveram o Adolescent Sedentary Activity Questionnaire (ASAQ) ${ }^{(13)}$, o qual foi adaptado transculturalmente e testado quanto à sua reprodutibilidade para o contexto brasileiro ${ }^{(14)}$. Esse questionário apresenta-se como uma alternativa para o entendimento do comportamento sedentário de adolescentes, incluindo, além do tempo de tela, aspectos sociais, educacionais, culturais e de transporte para a obtenção do tempo sedentário global do adolescente. No instrumento original, assim como na sua versão em português, não se testou a consistência interna do instrumento. Em sua versão em português, testou-se a sua reprodutibilidade em adolescentes de 12 a 17 anos, apresentando 
níveis elevados de Coeficiente de Correlação Intraclasse $(\mathrm{CCl})$ para o tempo total sedentário durante a semana $(\mathrm{CCl}=0,88 ; \mathrm{IC} 95 \%=0,82-0,91)$ e no fim de semana $(\mathrm{CCl}=0,77 ; \mathrm{IC} 95 \%=0,68-0,84)^{(14)}$.

Sendo assim, há a necessidade de se testar a reprodutibilidade e a consistência interna desse instrumento, tendo em vista que pode ser interpretado de forma diferente dependendo da idade e do contexto social no qual os escolares estão inseridos. Escolares de menor faixa etária ( 9 a 11 anos) podem ter uma menor compreensão das questões do questionário, o que pode diminuir sua confiabilidade.

Para testar se o questionário de comportamento sedentário ASAQ poderia ser utilizado tanto por crianças quanto por adolescentes, de diferentes regiões do Brasil, objetivou-se, no presente estudo, avaliar a fidedignidade de um questionário de comportamento sedentário.

\section{MÉTODOS}

Esta pesquisa trata-se de um estudo transversal, de caráter intencional, realizado com escolares de 9 a 15 anos, matriculados em escolas públicas, da cidade de Curitiba, Paraná, Brasil. De acordo com o Censo Escolar 2015, realizado pelo Instituto Nacional de Estudos e Pesquisas Educacionais Anísio Teixeira (INEP), 133.081 estudantes, de ambos os sexos, estavam matriculados no período diurno do ensino fundamental e médio de escolas estaduais e municipais da rede de ensino público da cidade ${ }^{(15)}$.

Para o desenvolvimento do presente estudo, realizou-se o cálculo do tamanho mínimo da amostra no programa GPower, versão 3.1.7. Considerando um tamanho de efeito de 0,20 , alpha $(\alpha)$ de 0,05 e poder de 0,95 , a amostra mínima necessária para a realização das análises propostas é de 314 escolares. Para a análise das informações, considerando a amplitude da faixa etária, optou-se em segmentar a amostra em três grupos, como sugerido pela $\mathrm{OMS}^{(7)}$ : 9 e 10 anos, 11 e 12 anos e 13 a 15 anos, o que resultou em uma amostra mínima final necessária de 942 escolares. No entanto, ao final do processo de coleta de dados, participaram deste estudo 1.119 escolares, de ambos os sexos, com idade entre 9 a 15 anos, totalizando 177 participantes a mais do que o mínimo calculado.

Para a coleta de dados, selecionaram-se de forma intencional sete escolas, três estaduais e quatro municipais da rede pública de ensino, sendo selecionadas duas turmas por ano de ensino de cada escola. Realizou-se o convite para todos os alunos dessas turmas a participarem da pesquisa.

Efetuou-se a coleta de dados nos meses de março a dezembro de 2015, por uma equipe treinada e composta por professores e acadêmicos do curso de Educação Física do Centro de Estudos de Atividade Física e Saúde (CEAFS) da Universidade Federal do Paraná (UFPR), supervisionada pela pesquisadora principal.

Inicialmente, solicitou-se a autorização das escolas para realização do estudo e, no dia anterior à coleta de dados, entregou-se aos alunos o Termo de Consentimento Livre e Esclarecido (TCLE), para os pais ou responsáveis autorizarem seus filhos a participarem do estudo, e o Termo de Assentimento Livre e Esclarecido (TALE) aos adolescentes acima de 12 anos, para assinarem concordando em participar do estudo. No dia estipulado para a coleta de dados, de posse desses documentos, os escolares responderam ao questionário em sala de aula, durante o horário normal de aula, a partir de orientações prévias do grupo de aplicação.

Participaram desta pesquisa somente estudantes entre 9 e 15 anos, sendo excluídos os escolares com idades $<9,0$ anos e $>15,9$ anos. Não participaram da pesquisa os escolares que não apresentaram o TCLE assinado pelos pais ou responsáveis, os adolescentes acima de 12 anos que não assinaram o TALE e os escolares que se negaram a participar da coleta de dados. Considerou-se perda amostral quando o escolar preencheu o questionário incorretamente ou desistiu da participação no estudo.

Para a caracterização da amostra estudada, os adolescentes responderam questões sobre aspectos sociodemográficos e econômicos: sexo, idade, trabalho, moradia, tipo de residência, classe econômica e escolaridade do pai e da mãe.

Utilizou-se o Critério de Classificação Econômica Brasil (CCEB) ${ }^{(16)}$ para determinar o nível socioeconômico, o qual contabiliza a quantidade de itens existentes na residência do participante. Os escolares responderam se apresentavam cada item da lista em seu domicílio e a sua quantidade, o grau de instrução do chefe da família ou do responsável que o sustenta, e a presença de serviço público (água encanada e rua pavimentada). Em virtude do tamanho da amostra em cada categoria, classificaram-se os escolares nas seguintes classes: A (a mais alta), $\mathrm{B} 1+\mathrm{B} 2, \mathrm{C} 1+\mathrm{C} 2$ e D/E (as mais baixas), agrupando-se a escolaridade do pai e da mãe em duas categorias: $\leq 8$ anos de estudo e $>8$ anos de estudo.

O Questionário de Atividades Sedentárias aos Adolescentes (QASA) ${ }^{(14)}$ é composto por 13 itens, divididos em cinco aspectos (tempo de tela, educacional, cultural, social e transporte), em que os participantes relatam o tempo 
gasto em atividades sedentárias em horas e/ou minutos durante cada dia da semana e durante um típico fim de semana.

Convidaram-se os alunos a recordar as suas atividades diárias e relatar as horas e os minutos que normalmente gastam em cada uma em dois momentos distintos. Explicaram-se algumas perguntas com mais detalhes, como as questões relacionadas ao uso do computador para o lazer, sendo enfatizada que essa atividade inclui leitura de WhatsApp, Messenger, Facebook, e-mail, chat e blogs nas redes sociais.

Em questões relacionadas ao transporte para a escola ou para a igreja nos fins de semana, enfatizou-se que esse item significa frequentar aulas ou reuniões da comunidade destinadas a atividades culturais e/ou religiosas. Sempre que os adolescentes marcavam duas atividades ao mesmo tempo, por exemplo, a leitura por prazer na frente da TV, era computada somente uma atividade, sem dobrar o tempo ${ }^{(14)}$.

A administração do questionário seguiu alguns procedimentos para evitar possíveis erros: a) a verificação de cada resposta dos alunos para esclarecer algumas questões, tais como: considerar que o tempo sedentário não pode extrapolar 24 horas por dia e pedir que os adolescentes lembrem o tempo de sono e tempo gasto em atividades físicas, de modo que a medição do tempo sedentário não seja superestimada; b) a análise de outros aspectos que poderiam promover erros no preenchimento do questionário, tais como: tempo excessivo gasto em apenas uma atividade; contagem dupla de atividades que podem ser realizadas simultaneamente; campos incompletos; atividades relacionadas com o transporte durante dias úteis; e c) enfatizar a importância de lembrar-se de uma semana típica e normal ao responder às perguntas ${ }^{(14)}$.

Analisou-se a fidedignidade por meio da reprodutibilidade teste-reteste e da consistência interna. Estimou-se a reprodutibilidade por meio do procedimento de medidas repetidas, com intervalo de sete dias entre as aplicações, utilizando-se procedimentos idênticos àqueles adotados na primeira aplicação ${ }^{(12,14)}$. Não se consideraram no estudo os adolescentes que não compareceram no dia e na hora agendados para o preenchimento da réplica do questionário.

Calculou-se o número de minutos gastos pelos adolescentes em cada atividade sedentária, assim como o tempo total (o produto da soma de todas as atividades sedentárias), para os dias de semana, para o fim de semana e para os cinco aspectos (tempo de tela, educacional, cultural, social e transporte). Além disso, estratificaram-se os resultados por faixa etária: 9 e 10 anos, 11 e 12 anos e 13 a 15 anos.

Para a descrição das variáveis estratificadas por faixa etária, utilizou-se a distribuição de frequências absoluta e relativa. Utilizou-se o coeficiente de correlação intraclasse $(\mathrm{CCl})$ para determinar a reprodutibilidade teste-reteste do questionário, e o diagrama de dispersão de Bland Altman para verificar a concordância absoluta entre as réplicas de aplicação do questionário. Verificou-se a consistência interna do instrumento por meio do alpha de Cronbach, considerando-se somente os questionários da primeira aplicação. Efetuaram-se as análises estatísticas no programa SPSS, versão 21.0 , adotando o nível de significância de $5 \%$.

O Comitê de Ética em Pesquisa com Seres Humanos da Universidade Federal do Paraná (Parecer 722.529) aprovou o presente estudo, estando de acordo com a Resolução do Conselho Nacional de Saúde (CNS) nº 466/2012.

\section{RESULTADOS}

Participaram do estudo 1.119 escolares, com idades entre 9 e 15 anos, sendo 50,4\% ( $n=564$ ) do sexo masculino e 49,6\% ( $n=555)$ do sexo feminino. A maioria dos escolares não trabalha ( $n=1064 ; 95,4 \%)$, mora com pai e mãe $(n=685 ; 61,2 \%)$, em casa ou sobrado $(n=1001 ; 89,5 \%)$, é de nível socioeconômico $B(n=622 ; 55,6 \%)$ e apresenta pai $(n=642 ; 57,4 \%)$ e mãe $(n=721 ; 64,4 \%)$ com mais de oito anos de estudo. A Tabela I apresenta as características sociodemográficas e econômicas dos escolares, de 9 a 15 anos, distribuídos por faixa etária.

A Tabela II apresenta os resultados da análise de reprodutibilidade testereteste total e estratificado por faixa etária. Os $\mathrm{CCl}$ apresentaram valores superiores a 0,70 . As variáveis educacionais e de transporte, nas idades de 11 e 12 anos, apresentaram CCI nos dias de semana de 0,67 e 0,59, e nos fins de semana de 0,56 e 0,52, respectivamente. Nas idades de 9 e 10 anos, no fim de semana, as variáveis cultural e social apresentaram CCl de 0,57 e 0,54 , respectivamente. A reprodutibilidade apresentou valores mais elevados nos dias de semana do que nos fins de semana.

A Tabela III apresenta os resultados da análise de consistência interna total e estratificados por faixa etária. Analisando a consistência interna do instrumento, verificou-se alpha de Cronbach superior a 0,70, sendo maior nos dias de semana do que nos fins de semana. Para o grupo de 9 a 10 anos, o alpha de Cronbach variou de 0,55 a 0,91; para o grupo de 11 a 12 anos, variou de 0,45 a 0,89; e para o grupo de 13 a 15 anos, variou de 0,44 a 0,87. 
Tabela I - Características sociodemográficas e econômicas dos escolares, de 9 a 15 anos, distribuídos por faixa etária. Curitiba, Paraná, Brasil, 2015.

\begin{tabular}{|c|c|c|c|c|c|c|c|c|}
\hline \multirow[b]{2}{*}{ Variável } & \multicolumn{2}{|c|}{ Todos } & \multicolumn{2}{|c|}{9 e 10 anos } & \multicolumn{2}{|c|}{1 e 12 anos } & \multicolumn{2}{|c|}{13 a 15 anos } \\
\hline & $\mathbf{n}$ & $\%$ & $\mathbf{n}$ & $\%$ & $\mathbf{n}$ & $\%$ & $\mathbf{n}$ & $\%$ \\
\hline Todos & 1119 & 100 & 321 & 28,7 & 347 & 31,0 & 451 & 40,3 \\
\hline \multicolumn{9}{|l|}{ Sexo } \\
\hline Masculino & 564 & 50,4 & 169 & 52,6 & 173 & 49,9 & 222 & 49,2 \\
\hline Feminino & 555 & 49,6 & 152 & 47,4 & 174 & 50,1 & 229 & 50,8 \\
\hline \multicolumn{9}{|l|}{ Trabalho* } \\
\hline $\operatorname{Sim}$ & 51 & 4,6 & 0 & 0 & 11 & 3,2 & 40 & 8,9 \\
\hline Não & 1064 & 95,4 & 321 & 100 & 334 & 96,8 & 409 & 91,1 \\
\hline \multicolumn{9}{|l|}{ Com quem mora } \\
\hline Com pai e mãe & 685 & 61,2 & 195 & 60,7 & 222 & 64,0 & 268 & 59,4 \\
\hline Com pai ou mãe & 360 & 32,2 & 102 & 31,8 & 97 & 28,0 & 161 & 35,7 \\
\hline Outros & 74 & 6,6 & 24 & 7,5 & 28 & 8,0 & 22 & 4,9 \\
\hline \multicolumn{9}{|l|}{ Tipo de residência** } \\
\hline Casa/sobrado & 1001 & 89,5 & 287 & 89,4 & 307 & 88,5 & 407 & 90,4 \\
\hline Apartamento/outro & 117 & 10,5 & 34 & 10,6 & 40 & 11,5 & 43 & 9,6 \\
\hline \multicolumn{9}{|l|}{ Classe econômica } \\
\hline$A$ & 147 & 13,1 & 34 & 10,6 & 46 & 13,3 & 67 & 14,9 \\
\hline $\mathrm{B} 1+\mathrm{B} 2$ & 622 & 55,6 & 163 & 50,8 & 212 & 61,1 & 247 & 54,8 \\
\hline $\mathrm{C} 1+\mathrm{C} 2$ & 335 & 29,9 & 120 & 37,4 & 84 & 24,2 & 131 & 29,0 \\
\hline $\mathrm{D} / \mathrm{E}$ & 15 & 1,4 & 4 & 1,2 & 5 & 1,4 & 6 & 1,3 \\
\hline \multicolumn{9}{|l|}{ Escolaridade do pai } \\
\hline$\leq 8$ anos de estudo & 477 & 42,6 & 170 & 53,0 & 126 & 36,3 & 181 & 40,1 \\
\hline$>8$ anos de estudo & 642 & 57,4 & 151 & 47,0 & 221 & 63,7 & 270 & 59,9 \\
\hline \multicolumn{9}{|l|}{ Escolaridade da mãe } \\
\hline$\leq 8$ anos de estudo & 398 & 35,6 & 141 & 43,9 & 103 & 29,7 & 154 & 34,1 \\
\hline$>8$ anos de estudo & 721 & 64,4 & 180 & 56,1 & 244 & 70,3 & 297 & 65,9 \\
\hline
\end{tabular}

n: número da amostra correspondente; *:4 participantes não apresentaram dados referentes à variável Trabalho; **: 1 participante não apresentou dado referente à variável Tipo de residência

Tabela II - Medidas de reprodutibilidade teste-reteste do questionário total e estratificado por faixa etária, considerando a medida contínua de comportamento sedentário. Curitiba, Paraná, Brasil, 2015.

\begin{tabular}{|c|c|c|c|c|}
\hline Variável & Total & 9 e 10 anos & $\begin{array}{l}\text { Idade Cronológica } \\
11 \text { e } 12 \text { anos }\end{array}$ & 13 a 15 anos \\
\hline & $\mathrm{CCl}(\mathrm{IC} 95 \%)$ & CCI (IC95\%) & $\mathrm{CCl}(\mathrm{IC95 \% )}$ & CCI (IC95\%) \\
\hline Toda semana & $0,92(0,91-0,93)$ & $0,91(0,88-0,92)$ & $0,91(0,89-0,93)$ & $0,93(0,91-0,94)$ \\
\hline Tempo de tela & $0,91(0,90-0,92)$ & $0,88(0,85-0,90)$ & $0,90(0,88-0,92)$ & $0,91(0,90-0,93)$ \\
\hline Educacional & $0,79(0,76-0,81)$ & $0,80(0,75-0,84)$ & $0,70(0,63-0,75)$ & $0,79(0,75-0,83)$ \\
\hline Cultural & $0,81(0,79-0,83)$ & $0,80(0,76-0,84)$ & $0,81(0,76-0,84)$ & $0,82(0,78-0,85)$ \\
\hline Social & $0,84(0,82-0,86)$ & $0,73(0,66-0,78)$ & $0,80(0,76-0,84)$ & $0,84(0,80-0,86)$ \\
\hline Transporte & $0,70(0,67-0,74)$ & $0,72(0,65-0,78)$ & $0,63(0,54-0,70)$ & $0,74(0,68-0,78)$ \\
\hline Dias de semana & $0,90(0,89-0,91)$ & $0,88(0,86-0,91)$ & $0,88(0,85-0,90)$ & $0,91(0,89-0,92)$ \\
\hline Tempo de tela & $0,88(0,87-0,89)$ & $0,86(0,82-0,89)$ & $0,87(0,84-0,89)$ & $0,88(0,86-0,90)$ \\
\hline Educacional & $0,77(0,74-0,79)$ & $0,79(0,73-0,83)$ & $0,67(0,59-0,73)$ & $0,76(0,71-0,80)$ \\
\hline Cultural & $0,76(0,74-0,79)$ & $0,79(0,74-0,83)$ & $0,77(0,72-0,82)$ & $0,74(0,69-0,79)$ \\
\hline Social & $0,80(0,78-0,82)$ & $0,68(0,60-0,74)$ & $0,79(0,74-0,83)$ & $0,79(0,74-0,82)$ \\
\hline Transporte & $0,65(0,60-0,69)$ & $0,62(0,53-0,70)$ & $0,59(0,49-0,67)$ & $0,71(0,65-0,76)$ \\
\hline Final de semana & $0,83(0,81-0,85)$ & $0,78(0,72-0,82)$ & $0,83(0,79-0,86)$ & $0,84(0,81-0,87)$ \\
\hline Tempo de tela & $0,83(0,81-0,85)$ & $0,74(0,68-0,79)$ & $0,84(0,80-0,87)$ & $0,84(0,81-0,87)$ \\
\hline Educacional & $0,64(0,60-0,68)$ & $0,71(0,64-0,77)$ & $0,56(0,46-0,64)$ & $0,64(0,57-0,70)$ \\
\hline Cultural & $0,70(0,66-0,73)$ & $0,57(0,46-0,65)$ & $0,73(0,67-0,78)$ & $0,73(0,68-0,78)$ \\
\hline Social & $0,71(0,68-0,75)$ & $0,54(0,43-0,63)$ & $0,67(0,59-0,73)$ & $0,75(0,70-0,79)$ \\
\hline Transporte & $0,61(0,56-0,65)$ & $0,67(0,59-0,74)$ & $0,52(0,41-0,61)$ & $0,60(0,52-0,67)$ \\
\hline
\end{tabular}

CCI: Coeficiente de Correlação Intraclasse; IC95\%: Intervalo de confiança a 95\% 
Tabela III - Medidas de consistência interna do questionário total e estratificado por faixa etária considerando a medida contínua de comportamento sedentário. Curitiba, Paraná, Brasil, 2015.

\begin{tabular}{lcccc}
\hline & Total & $\mathbf{9}$ e $\mathbf{1 0}$ anos & $\begin{array}{c}\text { Idade Cronológica } \\
\mathbf{1 1} \text { e } \mathbf{1 2} \text { anos }\end{array}$ & $\mathbf{1 3}$ a 15 anos \\
Variável & $\mathbf{\alpha}$ & $\mathbf{\alpha}$ & $\mathbf{\alpha}$ & $\mathbf{\alpha}$ \\
\hline Toda semana & $\mathbf{0 , 8 9}$ & $\mathbf{0 , 9 1}$ & $\mathbf{0 , 8 9}$ & 0,81 \\
Tempo de tela & 0,83 & 0,85 & 0,84 & 0,81 \\
Educacional & 0,82 & 0,85 & 0,81 & 0,80 \\
Cultural & 0,82 & 0,84 & 0,81 & 0,76 \\
Social & 0,76 & 0,79 & 0,74 & 0,84 \\
Transporte & 0,82 & 0,81 & 0,81 & $\mathbf{0 , 8 6}$ \\
Dias de semana & $\mathbf{0 , 8 7}$ & $\mathbf{0 , 8 9}$ & $\mathbf{0 , 8 7}$ & 0,80 \\
Tempo de tela & 0,82 & 0,83 & 0,81 & 0,80 \\
Educacional & 0,81 & 0,83 & 0,81 & 0,78 \\
Cultural & 0,79 & 0,80 & 0,80 & 0,82 \\
Social & 0,83 & 0,85 & 0,82 & 0,87 \\
Transporte & 0,86 & 0,85 & 0,85 & $\mathbf{0 , 7 1}$ \\
Final de semana & $\mathbf{0 , 7 4}$ & $\mathbf{0 , 7 7}$ & $\mathbf{0 , 7 3}$ & 0,60 \\
Tempo de tela & 0,62 & 0,65 & 0,61 & 0,57 \\
Educacional & 0,60 & 0,66 & 0,56 & 0,51 \\
Cultural & 0,55 & 0,56 & 0,56 & 0,44 \\
Social & 0,48 & 0,55 & 0,45 & 0,56 \\
Transporte & 0,55 & 0,58 & 0,52 & \\
\hline
\end{tabular}

a: alpha de Cronbach

A Figura 1 apresenta o diagrama de dispersão de Bland Altman para a concordância absoluta entre as duas aplicações do questionário. Analisando os minutos de comportamento sedentário total, incluindo dia de semana e fim de semana, observou-se uma diferença média entre as duas medidas de, aproximadamente, quatro horas, com intervalos de confiança variando de 175,86 a 280,68 . Os limites de concordância demonstram variabilidade na concordância entre as medidas da primeira e da segunda aplicação do questionário.

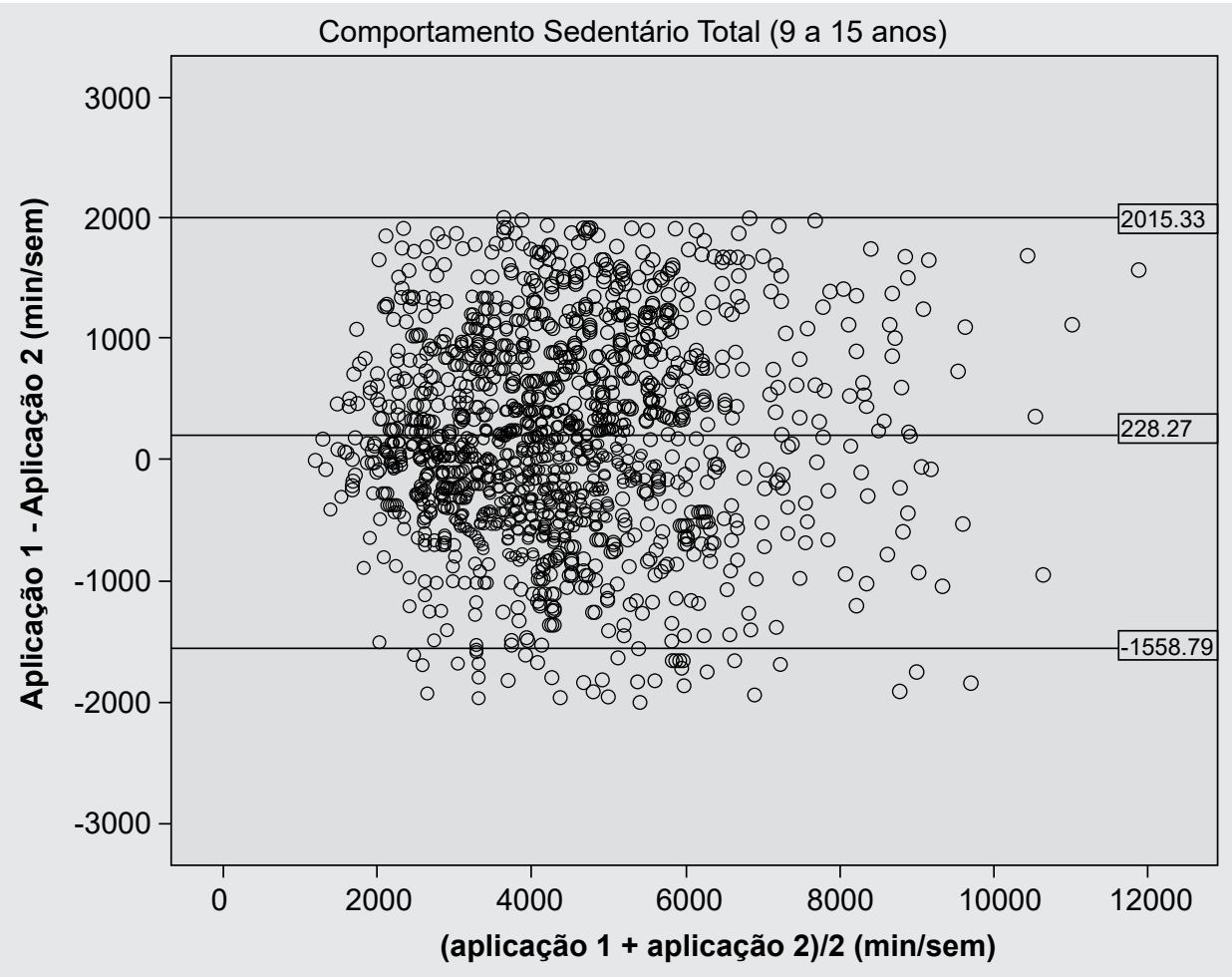

Figura 1 - Gráfico de Bland-Altman para verificar o grau de concordância entre as réplicas de aplicação - testereteste do questionário de comportamento sedentário dos escolares de 9 a 15 anos. Curitiba, Paraná, Brasil, 2015. 


\section{DISCUSSÃO}

O comportamento sedentário, principalmente o tempo prolongado sentado, representa um fator de risco em potencial para a saúde das pessoas, pois é positivamente associado ao aumento do risco de doenças cardiometabólicas, de mortalidade por todas as causas e de uma variedade de problemas fisiológicos e psicológicos ${ }^{(4,17)}$. Ele está relacionado a um estilo de vida não saudável na infância e na adolescência ${ }^{(4,17)}$.

Em uma revisão sistemática, autores examinaram a relação entre comportamento sedentário e indicadores de saúde em crianças e adolescentes, de 5 a 17 anos, e encontraram que o aumento de comportamento sedentário está associado ao maior risco cardiometabólico, à diminuição da aptidão física, aos baixos escores de autoestima e ao aumento do sobrepeso e da obesidade ${ }^{(18)}$.

No contexto de promoção da saúde na infância e na adolescência, o monitoramento adequado do comportamento sedentário apresenta-se necessário, tendo em vista a sua relação com fatores de risco à saúde, tanto na infância quanto na idade adulta ${ }^{(4,17)}$. Diante disso, a utilização de instrumentos confiáveis para avaliar o comportamento sedentário em crianças e adolescentes objetiva melhorar a qualidade das informações coletadas sobre esse comportamento de risco ${ }^{(12)}$, alvo do presente estudo.

O Questionário de Atividades Sedentárias para Adolescentes ${ }^{(13)}$ apresentou níveis satisfatórios de reprodutibilidade teste-reteste na atual pesquisa, com $\mathrm{CCl}$ acima de 0,70 . $\mathrm{CCl}$ menor do que 0,40 expressa baixa correlação; entre 0,40 e 0,75 , boa correlação, e maiores que 0,75 expressam excelente correlação ${ }^{(19)}$.

Há uma grande variação nos coeficientes de reprodutibilidade teste-reteste dos questionários de comportamento sedentário em adolescentes. Numa revisão sistemática, encontrou-se que o $\mathrm{CCl}$ para reprodutibilidade teste-reteste de questionários varia de 0,21 a 0,91. Tais discrepâncias podem ser atribuídas às diferenças nas idades dos escolares que participaram dos estudos, bem como às diferenças no intervalo das aplicações do teste e do reteste ${ }^{(12)}$.

O intervalo de sete dias entre as réplicas de aplicação do questionário pode influenciar nos níveis de reprodutibilidade. Utilizar intervalos prolongados entre as réplicas de aplicação do instrumento pode favorecer mudanças nas atividades praticadas, resultando em subestimação da reprodutibilidade. Por outro lado, caso o intervalo seja muito curto, pode-se ter o efeito memória, resultando em superestimação da reprodutibilidade do instrumento ${ }^{(13)}$.

O instrumento analisado no presente estudo apresentou aspectos psicométricos semelhantes aos instrumentos originais $^{(13,14)} \mathrm{e}$ aos resultados encontrados na literatura ${ }^{(20-22)}$, mostrando-se adequado para avaliação do comportamento sedentário em escolares de 9 a 15 anos. No atual estudo, os dados de reprodutibilidade apresentaram valores mais elevados nos dias de semana do que nos fins de semana. Esses resultados convergem com os estudos originais ${ }^{(13,14)}$.

Essa tendência se deve aos comportamentos de dia de semana serem caracterizados pela rotina escolar, de modo geral, e os fins de semana serem mais instáveis, ou seja, variarem sob a influência do tempo, de compromissos sociais, culturais e até educacionais, da possibilidade de passar mais tempo em frente a TV e ao computador, de condições climáticas e ainda da existência de oportunidades para a prática de atividade física ${ }^{(23)}$. Assim, na presente investigação, os valores de $\mathrm{CCl}$ mais elevados para os dias da semana em comparação com os dias de fim de semana sugerem variabilidade comportamental em vez de baixa confiabilidade no instrumento.

As variáveis educacionais e transporte, nas idades de 11 e 12 anos, apresentaram valores de $\mathrm{CCl}$ variando de 0,50 a 0,70. A variável transporte é uma limitação do estudo, já que uma única questão avalia o deslocamento sem diferenciar uma viagem mais longa de uma simples ida ao mercado ${ }^{(14)}$. No entanto, a variável educacional é avaliada por quatro questões, que podem variar muito de uma semana para outra a depender do período escolar e das exigências de cada escola.

No presente estudo, nas idades de 9 e 10 anos, as variáveis cultural e social no fim de semana apresentaram $\mathrm{CCl}$ variando de 0,50 a 0,60. A variável cultural é avaliada por três questões envolvendo leitura, artesanato e instrumento musical. Baixos coeficientes podem refletir características inerentes aos padrões dessas atividades que, diferentemente das atividades educacionais, não possuem rotina pré-estabelecida, podendo variar de uma semana para outra, tendo como base os interesses das crianças.

No que se refere à variável social, é composta por atividades como ficar à toa sem fazer nada, ter aulas em sala na escola aos sábados ou ir à igreja. $\mathrm{O}$ "ficar à toa" pode apresentar-se como uma variável difícil para a recordação precisa da criança, visto que pode compreender boa parte do tempo fora da escola. A tendência de queda dos valores de $\mathrm{CCl}$ converge com os achados dos estudos que utilizaram o mesmo instrumento em adolescentes, especialmente nos aspectos cultural, social e transporte ${ }^{(13,14)}$.

Os resultados visualizados no presente estudo para a consistência interna do instrumento apresentaram níveis satisfatórios $(>0,70)$, semelhantes aos resultados encontrados na literatura ${ }^{(24,25)}$. Um instrumento passa a ser 
considerado como tendo níveis adequados de consistência interna quando o valor de $\alpha$ for igual ou superior a $0,70^{(26)}$. Na presente investigação, o alpha de Cronbach apresentou menores valores nos fins de semana ( $\alpha=0,44$ a 0,77 ). Os escolares podem ter dificuldade para recordar as atividades realizadas no fim de semana por não apresentarem uma rotina pré-determinada, como acontece durante a semana, o que pode ter contribuído para os menores valores de consistência interna do questionário.

A análise de Bland-Altman realizada neste estudo demostrou variabilidade na concordância das respostas de acordo com o tempo de comportamento sedentário total de cada indivíduo. Esse resultado denota uma variabilidade natural do comportamento dos escolares nos dias de avaliação, bem como a dificuldade em recordar algumas atividades $^{(27)}$.

O IC 95\%, na análise de concordância realizada neste estudo, apresenta variações significativas que necessitam ser consideradas. No entanto, deve-se destacar que, mesmo com a diminuição da consistência das respostas do tempo total sedentário na maior parte dos casos, os valores estavam dentro do intervalo de confiança de $95 \%$, apresentando resultados semelhantes ao estudo original ${ }^{(14)}$. Não houve excesso de valores discordantes de elevada magnitude e a maior parte das diferenças individuais entre as duas medidas estavam dentro dos limites recomendados.

Destacam-se como pontos positivos deste estudo: o elevado tamanho amostral, com poder adequado para as análises propostas, bem como uma amostra proveniente de escolas estaduais e municipais, com diversidade etária, que proporcionou a avaliação tanto de crianças quanto de adolescentes. Em relação às limitações, destacase a falta de validação concorrente com um instrumento padrão ouro, como o uso do acelerômetro, e que medidas subjetivas podem ser influenciadas por respostas que são socialmente aceitas ou esperadas pelos grupos de crianças e adolescentes ${ }^{(28)}$. Além disso, como neste estudo os escolares recordaram o comportamento sedentário praticado em diferentes períodos (semanas distintas nas duas aplicações), mudanças ocorridas no comportamento sedentário nesse período podem influenciar nas medidas de reprodutibilidade.

Demonstrou-se, assim, a relevância de se investigar o comportamento sedentário de escolares para a promoção da saúde ${ }^{(4,5,9-12)}$, já que sua avaliação precisa é fundamental para monitorar as tendências desse comportamento, examinar seus efeitos nocivos sobre a saúde quando interfere na mortalidade, e ainda avaliar se as intervenções tem sido eficazes.

Sugere-se que novos estudos sejam realizados avaliando a validade concorrente do instrumento com a utilização da acelerometria.

\section{CONCLUSÃO}

O questionário analisado neste estudo apresentou fidedignidade satisfatória, podendo ser utilizado para mensurar o comportamento sedentário em escolares de 9 a 15 anos de escolas públicas.

\section{CONFLITOS DE INTERESSE}

Autores relatam a não existência de conflito de interesses.

\section{CONTRIBUIÇÕES}

Eliane Denise Araújo Bacil e Wagner de Campos contribuíram para o delineamento do estudo, a análise de dados, a escrita e a revisão final do manuscrito. Thiago Silva Piola contribuiu para a análise de dados, a escrita e a revisão final do manuscrito. Priscila lumi Watanabe, Michael Pereira da Silva e Rosimeide Francisco dos Santos Legnani contribuíram para a escrita e a revisão final do manuscrito.

\section{REFERENNCIAS}

1. Barnes J, Behrens TK, Benden ME, Biddle S, Bond D, Brassard P, et al. Letter to the editor: standardized use of the terms" sedentary" and" sedentary behaviours". Appl Physiol Nutr Metab. 2012;37(3):540-2.

2. Van Ekris E, Altenburg TM, Singh AS, Proper KI, Heymans MW, Chinapaw MJM. An evidence-update on the prospective relationship between childhood sedentary behaviour and biomedical health indicators: a systematic review and meta-analysis. Obes Rev. 2016;17(9):833-49. 
3. Biddle SJ, Garcia Bengoechea E, Wiesner G. Sedentary behaviour and adiposity in youth: a systematic review of reviews and analysis of causality. Int J Behav Nutr Phys Act. 2017;14(43):1-21.

4. Cliff DP, Hesketh KD, Vella SA, Hinkley T, Tsiros MD, Ridgers ND, et al. Objectively measured sedentary behaviour and health and development in children and adolescents: systematic review and meta-analysis. Obes Rev. 2016;17(4):330-44.

5. Rezende LFM, Sá TH, Mielke GI, Viscondi JYK, Rey-López JP, Garcia LMT. All-cause mortality attributable to sitting time: analysis of 54 countries worldwide. Am J Prev Med. 2016;51(2):253-63.

6. American Academy of Pediatrics, Committee on Public Education. American Academy of Pediatrics: children, adolescents, and television. Pediatrics. 2001;107(2):423-6.

7. World Health Organization. Growing up unequal: gender and socioeconomic differences in young people's health and well-being. Health Behaviour in School-aged children (HBSC) Study. Geneva: WHO; 2016 [acesso em 2017 Nov 11]. Disponível em: http://aventurasocial.com/arquivo/1458134965_HSBC-No.7-Growing-up-unequal-FULLREPORT.pdf

8. Instituto Brasileiro de Geografia e Estatística. Pesquisa Nacional de Saúde do Escolar 2015. Rio de Janeiro: IBGE; 2016 [acesso em 2017 Maio 22]. Disponível em: http://biblioteca.ibge.gov.br/visualizacao/livros/liv97870. pdf

9. Ishii K, Shibata A, Adachi M, Nonoue K, Oka K. Gender and grade differences in objectively measured physical activity and sedentary behavior patterns among japanese children and adolescents: a cross-sectional study. BMC Public Health. 2015;15(1254):1-9.

10. Ferreira RW, Rombaldi AJ, Ricardo LI, Hallal PC, Azevedo MR. Prevalence of sedentary behavior and its correlates among primary and secondary school students. Rev Paul Pediatr. 2016;34(1):56-63.

11. Lucena JM, Cheng LA, Cavalcante TL, Silva VA, Farias JC Junior. Prevalence of excessive screen time and associated factors in adolescents. Rev Paul Pediatr. 2015;33(4):407-14.

12. Hidding LM, Altenburg TM, Mokkink LB, Terwee CB, Chinapaw MJ. Systematic Review of Childhood Sedentary Behavior Questionnaires: what do we know and what is next? Sports Med. 2017;47(4):677-99.

13. Hardy LL, Booth ML, Okely AD. The reliability of the Adolescent Sedentary Activity Questionnaire (ASAQ). Prev Med. 2007;45(1):71-4.

14. Guimarães RF, Silva MPD, Legnani E, Mazzardo O, Campos W. Reproducibility of adolescent sedentary activity questionnaire (ASAQ) in Brazilian adolescents. Rev Bras Cineantropom Desempenho Hum. 2013;15(3):276-85.

15. Instituto Nacional de Estudos e Pesquisas Educacionais Anísio Teixeira. Censo Escolar 2015. 2016 [acesso em 2016 Mar 03]. Disponível em: http://matricula.educacenso.inep.gov.br/controller.php

16. Associação Brasileira de Empresas de Pesquisa. Critério de classificação econômica Brasil. 2015 [acesso em 2015 Jun 05]. Disponível em: http://www.abep.org/criterio-brasil.

17. Ekelund U, Luan J, Sherar LB, Esliger DW, Griew P, Cooper A. Moderate to vigorous physical activity and sedentary time and cardiometabolic risk factors in children and adolescents. JAMA. 2012;307(7):704-12.

18. Carson V, Hunter S, Kuzik N, Gray CE, Poitras VJ, Chaput JP, et al. Systematic review of sedentary behaviour and health indicators in school-aged children and youth: an update. Appl Physiol Nutr Metab. 2016;41(6 Suppl 3):S240-65.

19. Landis JR, Koch GG. The measurement of observer agreement for categorical data. Biometrics. 1977;33(1):15974.

20. Kozey Keadle S, Lyden K, Hickey A, Ray EL, Fowke JH, Freedson PS, et al. Validation of a previous day recall for measuring the location and purpose of active and sedentary behaviors compared to direct observation. Int $\mathrm{J}$ Behav Nutr Phys Act. 2014;11(12):1-11.

21. Cabanas-Sanchez V, Martinez-Gomez D, Esteban-Cornejo I, Castro-Pinero J, Conde-Caveda J, Veiga OL. Reliability and validity of the Youth Leisure-time Sedentary Behavior Questionnaire (YLSBQ). J Sci Med Sport. 2018;21(1):69-74. 
22. Farias JC Júnior, Loch MR, Lima AJ Neto, Sales JM, Ferreira FELL. Reprodutibilidade, consistência interna e validade de construto do KIDSCREEN-27 em adolescentes brasileiros. Cad Saude Publica. 2017;33(9):1-14.

23. Jago R, Anderson CB, Baranowski T, Watson K. Adolescent patterns of physical activity: differences by gender, day, and time of day. Am J Prev Med. 2005;28(5):447-52.

24. Han H, Gabriel KP, Kohl HW. Evaluations of validity and reliability of a transtheoretical model for sedentary behavior among college students. Am J Health Behav. 2015;39(5):601-9.

25. Huang YJ, Wong SH, Salmon J, Hui SS. Reliability and validity of psychosocial and environmental correlates measures of physical activity and screen-based behaviors among Chinese children in Hong Kong. Int J Behav Nutr Phys Act. 2011;8:16.

26. Field A. Descobrindo a estatística usando SPSS. Porto Alegre: Artmed; 2009.

27. Kohl HW, Fulton JE, Caspersen CJ. Assessment of physical activity among children and adolescents: a review and synthesis. Prev Med. 2000;31(2):54-76.

28. Corder K, Ekelund U, Steele RM, Wareham NJ, Brage S. Assessment of physical activity in youth. J Appl Physiol. 2008;105(3):977-87.

\section{Endereço para correspondência:}

Eliane Denise Araújo Bacil

Universidade Federal do Paraná - UFPR

Centro de Estudos em Atividade Física e Saúde - CEAFS

Rua Coração de Maria, 92

Bairro: Jardim Botânico

CEP: 80210170 - Curitiba - PR - Brasil

E-mail: elianebacil@hotmail.com 